http://doi.org/10.15359/ree.14-1.6

\title{
Consideraciones básicas del pensamiento complejo de Edgar Morin, en la educación
}

\author{
José Miguel Pereira Chaves ${ }^{l}$ \\ Académico de la Escuela de Ciencias Biológicas, Universidad Nacional \\ Heredia, Costa Rica
}

Recibido 18 de noviembre de 2009 • Aceptado 02 de diciembre de 2009

Resumen. Este ensayo consiste en una breve descripción y un análisis del pensamiento complejo de Edgar Morin; para ello se hace mención al enfoque sistémico el cual se refiere a la interconexión y a las interacciones entre los objetos, las personas y el ambiente como un todo, lo cual sirve de fundamento para la propuesta educativa, en la que el conocimiento se debe abordar de manera integral y no fragmentado. Por lo anterior, el conocimiento humano debe fomentar la toma de conciencia de que somos tan sólo una parte componente de un sistema más general (complejo, y en constante interacción), en el que la educación puede ser el medio efectivo para que los alumnos desarrollen la capacidad de comprender otros sistemas complejos, además de los seres vivos. Aquí cobran importante interés las "nuevas ciencias" y las humanidades.

Palabras clave. Pensamiento, complejidad, conocimiento, educación, sistema.

Abstract. This essay consists of a brief description and analysis of Edgar Morin's complex thinking. For that reason, it focuses on the systematic approach that refers to the interconnection and interactions of objects, people, and environment as a whole, which serve as the basis of the educational proposal, where knowledge must be regarded as an integrated fashion and not as something fragmented. Thus, human knowledge should raise awareness that we are just a component of a more general system (complex and in constant interaction), where education can be an effective source for students to develop the capacity of understanding other complex systems, besides living organisms, where also there is a big influence from "new sciences" and humanities. Therefore, the purpose of this work is to develop those ideas proposed by Edgar Morin.

Key words. Thinking, complexity, knowledge, education, system.

${ }^{1}$ Coordinador de las Olimpiadas Costarricense de Ciencias Biológicas e internacionales y académico del área de Enseñanza de las Ciencias, y de la Comisión de trabajos finales de graduación de la Maestría en Ciencias Marinas de la Escuela de Ciencias Biológicas, Universidad Nacional, Heredia, Costa Rica. Licenciatura en Ciencias de la Educación con énfasis en Didáctica de las Ciencias, 2003. Maestría en Ciencias Marinas, 2007. Estudiante del Programa Doctoral en Educación de la Universidad Estatal a Distancia. Apartado 86-3000. Correo electrónico: jpereira@una.ac.cr

La Revista Electrónic@ Educare está indizada en el Catálogo LATINDEX.

Artículo protegido por la licencia Creative Commons. 


\section{Introducción}

Desde la perspectiva del pensamiento complejo y estrechamente relacionado con la posición que, dentro de las Ciencias Sociales, defiende el denominado "enfoque sistémico" -ningún objeto o acontecimiento (que deba ser analizado por la ciencia) se encuentra aislado o desvinculado, sino que éste aparece dentro de un sistema complejo, desde donde entabla una gama de relaciones con otros objetos, ya sea "internos" (con otros objetos componentes del sistema), o "externos" (con elementos del ambiente, del entorno o del contexto de ese sistema). Ésta es la tesis que, en términos generales, sirve de fundamento para la propuesta educativa de Edgar Morin.

A grandes rasgos, esta propuesta intenta integrar el enfoque global y complejo del mundo con una educación que tome en cuenta esta visión. Así, todo conocimiento humano que nos permita tomar conciencia de que somos tan sólo una parte componente de un sistema más general (complejo y en constante interacción), será bienvenido para formar parte de la educación. En esta propuesta se despierta un gran interés por las "nuevas ciencias" y las humanidades. Sin embargo, no es ésta una tarea sencilla: ello implica derribar los esquemas mentales que, desde el comienzo de la ciencia moderna, nos han sido impuestos (entre ellos: la visión de un mundo fragmentario y mecánico). Por ello, desde esta perspectiva se habla, no sólo de una nueva educación, sino de una "reforma del pensamiento". Este ensayo tiene como propósito desarrollar estas ideas expuestas por Morin. Un primer y breve apartado se dedica a la presentación biográfica del autor.

\section{Edgar Morin: una breve presentación}

Edgar Morin es un reconocido filósofo y político francés nacido en 1921. Durante sus años de juventud, estuvo vinculado con movimientos socialistas y comunistas de su país, relación que le puso en contacto con textos y teorías marxistas. En 1951, es expulsado del Partido Comunista y, en ese mismo año, se integra al Centro Nacional de Investigación Científica (CNRS), donde inicia su estudio en matemática así como una relación con movimientos artísticos tales como el surrealismo.

Durante esos años, funda y dirige la revista Argumentos. En la década de los 60's, Morin emprende una serie de viajes a Latinoamérica e inicia su estudio en problemáticas que intenten explicar el desarrollo del sujeto humano. Ya para la década de los 70's, y con el nacimiento y el impulso de las biotecnologías (así como con la aparición de la teoría de sistemas, la cibernética y la teoría de la información), Morin desarrolla sus ideas, las cuales desembocan en su propuesta epistemológica de la complejidad.

\section{El pensamiento complejo y la fragmentación del conocimiento}

El enfoque del pensamiento complejo parte de la idea de que cualquier elemento del mundo no es un objeto aislado, sino que forma parte de un sistema mayor que lo contiene, por lo que se encuentra en constante interacción con otros elementos del sistema, así como con el sistema completo. Desde este enfoque, las sociedades, los individuos, incluso el universo se consideran "sistemas complejos", sujetos a múltiples relaciones e interacciones entre sus componentes y con otros sistemas. Así, dentro de esta perspectiva sistémica (o dentro de lo que hemos denominado "epistemología de la complejidad"), se parte del hecho de que en todo grupo humano estructurado 
(desde la familia y amigos, hasta el sistema mundial), sus componentes (los seres humanos) se encuentran estrechamente vinculados, entre sí y con el ambiente, por lazos de tipo biológico, económico, espiritual, político, cultural, etc.

La epistemología de la complejidad supone, pues, una interacción entre seres humanos (unos con otros) y entre seres humanos y medio ambiente (contexto), interacción sin la cual resulta imposible comprender nuestro lugar y nuestro papel en el universo. Ésta es una tesis central defendida por el enfoque de la complejidad, la cual, en el planteamiento de Morin, implica importantes inferencias. Según este autor, la falta de la "visión global" (producto del pensamiento complejo) implica la pérdida de conciencia respecto de nuestra verdadera condición humana, es decir, nuestra condición de personas que habitan un mundo y que mantienen lazos afectivos, espirituales, sociales, económicos, etc., con otros.

Según Morin, nuestra vida supone una multiplicidad de relaciones (así como una diversidad de ámbitos en los que se desenvuelve). Tomar conciencia de esa multiplicidad es lo que nos permite desarrollarnos como sujetos humanos (en un sentido integral del término) y no sólo como simples objetos. Así, una visión diferente del mundo (basada en el pensamiento complejo) supone un cambio en la manera en que afrontamos la vida. Sin embargo, la visión de mundo defendida por el pensamiento de la complejidad no ha sido la que ha prevalecido en la historia de occidente (o al menos desde el inicio de la modernidad).

Para Morin, la supremacía de la racionalidad científica moderna (analítica, mecánica y reduccionista) tuvo como catastrófico resultado la creación de una visión del mundo "simplificada" y mecánica. Como consecuencia de la aplicación del método analítico, las ciencias crearon la visión del mundo en la que los objetos "simples" y aislados constituyen lo esencial, y en la que las relaciones y la interacción pasan a un segundo plano. Así, la ciencia moderna, con sus métodos de investigación, nos han hecho creer que el mundo es tan sólo "la suma de las partes" y no un conjunto complejo de múltiples elementos en constante interacción.

Ésta es la visión de mundo que combate Morin (visión de mundo producto de la racionalidad científica tradicional). Sin embargo, es importante una aclaración: no puede afirmarse que Morin niegue que el conocimiento humano (reflejado en la ciencia y la tecnología) haya generado cambios, más o menos positivos, a las sociedades. Morin insiste, sobre todo, en que estos conocimientos han llegado, a lo largo de los siglos, al extremo de borrar y desconocer la naturaleza misma de aquello que estudia: en el marco de las especializaciones disciplinarias en el transcurso del siglo XX (especialización que se origina ya desde el inicio de la modernidad), se ha perdido la perspectiva de complejidad, multiplicidad y prolijidad de los factores que intervienen en la constitución de cada elemento en el universo (ya sea que hablemos de "elementos" sociales, físicos, biológicos u otros), así como su vínculo con las diferentes "partes" de los sistemas a los que pertenecen.

Estos progresos científicos han sido dispersos y aislados a causa de, precisamente, la especialización absoluta de dicho conocimiento y a su afán, dada esa peculiaridad, de evitar el contexto y las globalidades como aspectos esenciales de su análisis. Así, las realidades globales (complejas) se han quebrantado, y lo humano se ha desarticulado: sus múltiples dimensiones (biológica, social, cultural, económica, física, histórica, etc.) se han esparcido en una serie de saberes que muy pocas veces tienen interrelación entre sí. Éste ha sido, según Morin, el legado de la modernidad y de los enfoques utilitaristas del conocimiento que, desde Descartes, Bacon y, hasta, Marx han predominado en el ambiente científico y académico.

Éste no es un problema menor, sino que posee la mayor trascendencia, pues de esta tradición centenaria (y su visión simplificada del mundo) devienen, según Morin, los principales problemas que, actualmente, intenta combatir el denominado "pensamiento de la complejidad". Según esta 
perspectiva, resulta evidente que, bajo las condiciones impuestas por el conocimiento fragmentario, las personas han perdido su capacidad para contextualizar los saberes, es decir, su capacidad para integrarlos a los conjuntos (o sistemas) más amplios y complejos a los que pertenecen ${ }^{2} \mathrm{y}$, con ello, han perdido la verdadera noción de lo que representa la condición humana, la cual, para Morin, es una noción que contempla lo humano en tanto que "es a la vez físico, biológico, psíquico, cultural, social e histórico, es decir, una identidad compleja y común a todos los demás humanos" (Morin, 1999, p. 3).

En este sentido, surge, entonces, la necesidad de desarrollar la aptitud de las personas para ubicar el conocimiento y la información en un contexto y dentro de un conjunto. Para Morin, resulta imperativo concebir una educación que rompa con la visión fragmentaria del mundo, para, de esta forma, dar paso a una educación que enseñe los métodos que nos permitan "aprehender las relaciones mutuas y las influencias recíprocas entre las partes y el todo de un mundo complejo" (Morin, 1999, p. 2) y, así, vivir de acuerdo con nuestra (compleja) condición. Por tanto, la interrelación y la interacción son parte esencial de la perspectiva de Morin, en contraposición al enfoque analítico y fragmentario de las ciencias que se originaron en la modernidad.

Como ejemplos de nuevas ciencias o ciencias transdisciplinares están, en la línea de Morin, para las Ciencias sistémicas las siguientes: ecología, ciencias de la tierra, ciencias de gestión. En otra línea, Joël de Rosnay incluye nuevas ciencias como la Neobiología, consagradas al estudio de la vida artificial, y la Simbionomía, encargada de estudiar las conexiones y continuidades entre los fenómenos naturales y artificiales, artísticos y técnicos, culturales y civilizatorios en un complejo cognoscitivo coherente, es decir, un marco teórico global que abarquen las demás disciplinas.

\section{Pensamiento complejo y educación}

El caso de la fragmentación (descontextualización) de los saberes, no sólo impide el desarrollo de un verdadero conocimiento científico, sino que ello produce, en el campo de la educación, consecuencias más dañinas aún. Para Morin, el debilitamiento de la percepción de lo global conduce al debilitamiento del sentido de la responsabilidad y de la solidaridad que los seres humanos tenemos para con nuestro entorno. Sometidas a este paradigma fragmentario (y autoconsideradas como sujetos aislados), las personas no reflexionan sobre sus vínculos con los demás miembros de su comunidad, es decir, no reflexionan sobre su verdadera relevancia como partes de un sistema más general (como por ejemplo, la sociedad, el planeta e, incluso, el universo en su totalidad), al cual se deben (en tanto individuos). Éste es un problema que trasciende la realidad académica, permea nuestros actos sociales y deriva en consecuencias sumamente perjudiciales.

Respecto al compromiso ético-político y la tarea de la educación, en este contexto, afirma Morin (1999):

De allí [es decir, de esta problemática], se esbozan las dos grandes finalidades ético-políticas del nuevo milenio: establecer una relación de control mutuo entre la sociedad y los individuos por medio de la democracia y concebir la Humanidad como comunidad planetaria. La educación debe no sólo contribuir a una toma de conciencia de nuestra Tierra-Patria, sino también permitir que esta conciencia se traduzca en la voluntad de realizar la ciudadanía terrenal. (p. 7) ${ }^{2}$ Para Morin, "la supremacía de un conocimiento fragmentado según las disciplinas impide, a menudo, operar el vínculo entre las partes y las
totalidades" (Morin, 1999, p. 2). 
Así, la visión fragmentaria nos ha puesto de frente, por un lado -tal y como hemos dichoal constante interés individualista (muy de moda en nuestras sociedades) que sacrifica el interés común frente a los beneficios individuales. A expensas de otros seres humanos, las personas quieren poseer cada vez más, se desentienden de las necesidades sociales, mundiales, universales (a veces en franca oposición respecto de su interés privado) y, lo que es peor aún: nuestra falta de percepción de lo global nos ha hecho perder, a la vez, nuestro sentido de responsabilidad para con el planeta, lo que pone en riesgo nuestra supervivencia y la de muchas otras especies. El actual desastre ecológico es producto de una visión de mundo que no contempla nuestro vínculo con el ambiente que nos rodea, un vínculo frágil y que pone de manifiesto la interdependencia de todos los seres del planeta.

Ante esta problemática surge, entonces, la importancia de una educación que refleje, realmente, el mundo global y complejo en el que vivimos. Una educación que tome en cuenta esta perspectiva sistémica, abordará las necesidades y problemáticas humanas desde su verdadera dimensión: valorará la multiplicidad de elementos constitutivos que nos convierten en seres humanos, así como la diversidad de relaciones que tenemos unos con otros, con el resto de las especies y con el mundo en general (esto evaluará nuestra verdadera condición humana). No se trata, sin embargo, de una educación completamente nueva, desvinculada de los progresos y avances que han experimentado las diversas ciencias y los distintos saberes. Morin acude a los recursos y a los métodos de análisis que ya tenemos a disposición para plantear su reforma educativa. Por ello, da especial énfasis al aporte que, dada esta nueva visión "global y sistémica", pueden brindar las diversas áreas del conocimiento humano.

De este modo, nos dice, que la cultura científica ha desarrollado (recientemente) campos de estudio que ponen en evidencia la importancia de la epistemología de la complejidad. Tales son los casos, por ejemplo, de la cosmología y de la teoría de la evolución junto con la ecología ${ }^{3}$. Los nuevos enfoques cosmológicos nos han permitido comprender, por ejemplo, las complejas relaciones físicas que entrelazan cada elemento del universo (los átomos, las moléculas, etc.), así como el modo en que estas relaciones nos unen con el cosmos que nos rodea (y no sólo en el presente, sino ya desde el origen mismo del Universo).

La ecología, por su parte, al estudiar los seres vivos no en aislamiento, sino en su ambiente y en su interacción (entre sí y con el ambiente, en general), nos pone de manifiesto que la "visión integradora" (visión que analiza a cada elemento en tanto se encuentre sujeto a un contexto) se hace cada vez más necesaria, si queremos dar cuenta de los fenómenos (y de nuestra condición humana). El mismo conocimiento científico ha dado paso a esta nueva visión global, compleja y sistémica de los eventos del mundo.

Morin sugiere que el desarrollo del conocimiento científico debería integrarse en un currículum coherente dentro de los diversos niveles educativos (primaria, secundaria y universidad), de tal modo que el "enfoque sistémico" predomine en los programas de estudio. Así, por ejemplo, la teoría evolutiva podría plantear, en primaria, el problema de la emergencia del Homo Sapiens, de la cultura, del lenguaje, del pensamiento, lo que permitiría enlazar estos conocimientos, a su vez, con la psicología y la sociología (Morin, 2007). De esta forma, podría integrarse el surgimiento de la condición humana con la interrogación sobre el mundo. Para Morin, esta integración del conocimiento deberá fomentarse hasta la educación universitaria, de modo que, en esas casas de estudio, se abandone el viejo paradigma de la división de escuelas y facultades y se promueva la creación de nuevas unidades académicas mucho más

${ }^{3}$ Éstos son sólo dos ejemplos que nos permiten exponer la idea de Morin; sin embargo, el autor señala áreas generales del conocimiento científico que pueden colaborar en este propósito: la física cuántica, las ciencias naturales "renovadas" y las ciencias de la Tierra (Morin, 2007). Más adelante expondremos algunos ejemplos que, al respecto, sugiere Morin. 
globales (al respecto, Morin propone, por ejemplo, la creación de la Facultad de lo Humano o la Facultad de la Vida) ${ }^{4}$.

Pero no sólo la ciencia puede brindar herramientas de análisis para la epistemología de la complejidad y el enfoque educativo que persigue. La cultura de las humanidades cumple un papel muy importante en esta tarea. Para Morin, las humanidades (las artes, la literatura y la filosofía) nos permiten ver las complejas relaciones del ser humano con el otro, con la sociedad y con el mundo, las "inestabilidades del yo", la dimensión estética de la existencia, etc., es decir, aquellos aspectos que nos manifiestan nuestra verdadera condición. En otras palabras: ellas nos sensibilizan frente al amor y al dolor ajeno. Así, Morin nos habla de ejemplos importantes dentro de las humanidades que sirven de herramientas para la nueva educación (producto de la visión de la complejidad). Un caso especial es el de la novela, pues:

...ella nos transporta a través de la historia y de los continentes... nos revela la universalidad de la condición humana sumergiéndose en la singularidad de los destinos individuales localizados en el tiempo y en el espacio... nos deja ver que el ser más nimio tiene varias vidas, cumple varios papeles, vive en una existencia en parte de fantasmas, en parte de actos (Morin, 2007, pp. 46-47).

Esto nos conduce a otro aspecto importante dentro de este enfoque: la perspectiva sistémica se caracteriza por ser interdisciplinaria. Dada la gran variedad de factores que intervienen en la dinámica y en el funcionamiento de estas totalidades complejas (los sistemas sociales), así como de la diversidad cualitativa de las partes que la componen, se hace indispensable un abordaje desde distintas perspectivas (arte, economía, sociología, biología, etc.) de esos sistemas. No se trata, sin embargo, de un abordaje que produzca una división tajante entre cada una de estas áreas (como ha sucedido, reiteradamente, a lo largo de la historia), sino más bien de una forma de afrontar el estudio científico (y las políticas y prácticas que de él se derivan) de manera integral, para que, asimismo, la educación pueda desarrollar, de forma integral, a las personas.

Así, la enseñanza puede intentar que converjan las ciencias naturales, las ciencias humanas y la cultura de las humanidades en el estudio de la condición humana, de modo que ésta pueda desembocar en una toma de conciencia del destino común (conforme a un mundo global y complejo) que comparten todas las personas, conciencia que es propia de la "era planetaria" (la cual nos muestra que confrontamos, no como individuos sino como humanidad, los mismos problemas vitales).

Como hemos dicho, esta integración del conocimiento deberá contemplar los distintos niveles de la educación. Es en este sentido humanista e integral, que Morin propone su reforma educativa, una reforma que, básicamente, “...dé cuenta de que el conocimiento de las partes depende del conocimiento del todo" y que, a su vez, este conocimiento del todo depende del conocimiento de las partes (Morin, 2007, p. 92), es decir, que dé cuenta de la complejidad de las relaciones que entran en juego en la vida y en el mundo que habitamos. En el campo de la educación, ello implica que:

${ }^{4}$ Asimismo, Morin enfatiza la idea de que para la educación lo más importante es la transmisión de "una cultura que permita comprender nuestra condición y ayudarnos a vivir" y, en ese sentido, una educación que favorezca un modo "abierto y libre" de pensar (Morin, 2007: 11)

\footnotetext{
${ }^{5}$ Morin también tiene palabras de elogio para la filosofía: "La enseñanza de la filosofía podría revitalizarse para el aprendizaje de la vida. Entonces, podría proporcionar, a modo de viático, los dos productos más preciados de la cultura europea: la racionalidad crítica y autocrítica que, justamente, permite auto observarse y permite la lucidez y... la fe incierta. De esta manera, la filosofía volvería a encontrar su gran y profunda misión al contribuir a la conciencia de la condición humana y al aprendizaje de la vida" (Morin, 2007, p. 57)
} 
Hay que enseñar que las cosas no son solamente cosas, sino también sistemas que constituyen una unidad que vincula partes diversas; no objetos cerrados, sino entidades inseparablemente unidas a su entorno y que sólo pueden ser conocidas si se las inserta en su contexto. En lo que respecta a los seres vivos, éstos se comunican entre sí y con su entorno y estas comunicaciones forman parte de su organización y de su naturaleza. (Morin, 2007, p. 81)

Por ello, en el planteamiento de Morin no es posible hablar tan sólo de un cambio en los contenidos o los programas educativos (aunque, sin duda, esto es parte importante de la "reforma" en educación), sino de un cambio respecto del paradigma científico moderno de la fragmentación y la especialización: se trata, en última instancia, de una reforma del pensamiento que derribe los antiguos esquemas mentales, es decir, una reforma que implica tomar conciencia de nuestra condición humana, entendida como una condición que es producto de las complejas relaciones que tenemos con otras personas (y con el medio ambiente, en general). Así, según Morin (2007), se trata “...de una reforma que concierne nuestra aptitud para organizar el conocimiento, es decir, para pensar" (p. 88). De este modo, tomando como fundamento el principio de que "...el conocimiento de las partes depende del conocimiento del todo" (y viceversa), Morin (2004) propondrá los puntos indispensables de su reforma y los principios que sirven de guía para un pensamiento vinculante:

1. El principio sistémico u organizativo. Se trata del principio rector mencionado anteriormente, es decir, la unión del conocimiento de las partes con el conocimiento del todo, cuyo objetivo consiste en combatir la idea (reduccionista) de que el todo es tan sólo la suma de las partes.

2. El principio holográmico. Es decir, poner de manifiesto aquello presente en toda organización compleja: la parte está en el todo y, a su vez, el todo está inscrito en la parte. Por ejemplo: la célula es una parte de un todo (el organismo), pero, a la vez, la totalidad del patrimonio genético está contenido en cada célula individual.

3. El principio del bucle retroactivo o realimentación. Aquel que permite el conocimiento de los procesos autorregulados (feedback) y que rompe con el principio de la causalidad lineal. Por ejemplo: hace referencia al procesamiento de la información que le permite a la máquina o al sistema vivo regular su comportamiento, de acuerdo con su funcionamiento real y no en relación con lo que se espera, es un ir y venir permanente de la información, que actualiza esa información y le permite a la máquina o al organismo comportarse de acuerdo con propósitos actualizados.

4. El principio del bucle recursivo. Consiste en "un bucle generador", en el cual los efectos y los productos son, asimismo, productores y causantes de lo que los produce. Por ejemplo: los seres humanos somos producto de un sistema de reproducción (ancestral), pero este sistema no puede reproducirse al menos que nosotros nos convirtamos en productores al "acoplarnos".

5. El principio de autonomía/dependencia. El principio que dice que los seres vivos desarrollan su autonomía en dependencia de su ambiente (en el caso de los seres humanos, de su cultura).

6. El principio dialógico. Permite asumir, racionalmente, la inseparabilidad de nociones contradictorias para concebir un mismo fenómeno complejo. Por ejemplo: cuando se considera la especie o la sociedad, el individuo desaparece, pero cuando se considera al individuo es la sociedad la que desaparece. Según este principio, "el pensamiento debe asumir dialógicamente los dos términos que tienden a excluirse entre sî" (Morin, 2002). 
7. El principio de reintroducción del que conoce en todo conocimiento. Indica que todo conocimiento es una reconstrucción/traducción que una mente/cerebro hace en una cultura y un tiempo determinados.

Así, para Morin, la reforma del pensamiento constituye un intento por organizar el conocimiento de manera distinta, pero no como una simple (re)organización de los contenidos curriculares en los distintos niveles de la educación, sino como un modo de tener "la cabeza bien puesta", es decir, un modo de instaurar nuestras ideas bajo el paradigma de la complejidad, un paradigma que enfatiza en las complejas relaciones que nos constituyen como sujetos humanos. Ese es el sentido de los principios que Morin señala como indispensables para alcanzar este objetivo de la educación.

\section{Conclusiones}

Dentro de la perspectiva sistémica, partimos del hecho de que, cuando el científico social (y el educador) se preocupan por analizar y comprender las realidades a las que se enfrentan, no deberían segmentar y estudiar cada uno de estos subsistemas por separado (o como si no tuvieran relación uno con otro), pues se encontraría tan sólo con acontecimientos y evidencia aislada que no podría explicar la complejidad y la riqueza de las sistemas sociales de los seres humanos. Cuando fragmentamos y dividimos el conocimiento obtenemos una visión fragmentada y sesgada de la realidad. Éste es el paradigma mental combatido por Morin.

La tarea del pensamiento de la complejidad consiste en revelar la naturaleza compleja de los fenómenos. En esta labor, se encuentra implícito nuestro compromiso para con el mundo. Nos hallamos en una era planetaria. Así, debemos ligar la enseñanza de la condición humana con la ética de la comprensión entre las personas. La reforma del pensamiento debe estar al servicio de la humanidad entera, y, de esta manera, fomentar la solidaridad y la moral de la humanidad por sobre el individualismo y la destrucción.

En esta tarea ética, el pensamiento de la complejidad asume una posición activa: a partir de la toma de conciencia de que somos tan sólo una parte componente de un sistema más general, el enfoque sistémico supone que debemos actuar para estar en armonía con dicho sistema. Las nuevas ciencias y las humanidades cobran un valor especial en este contexto, pues ellas nos ayudan a comprender mejor nuestro lugar en el mundo. Se trata, en última instancia, de una reforma de nuestros esquemas mentales, de la manera en que pensamos y concebimos la inteligencia. Aquí se plantea el carácter político conflictivo entre las nuevas ciencias de la complejidad que son apoyadas y dirigidas por los grupos dominantes y las nuevas ciencias de la complejidad, que tienen orientación crítica y que buscan realizar los valores de la libertad, la democracia y la igualdad.

El paradigma de la complejidad desde la educación es indispensable abordarlo y entenderlo porque en el quehacer educativo no siempre está presente el conocimiento de la complejidad de la integración de saberes y de las reacciones o efectos que se dan en la multidisciplinariedad, la transversalidad y la multidimensionalidad, desde los distintos ámbitos. Por este motivo, dentro de esta situación se hacen invisibles los conjuntos complejos de interacción entre las partes y el todo, partiendo del desafío educativo como la integración del conocimiento en cada una de las partes para una mejor comprensión de la globalidad educativa.

La comprensión de la complejidad en el ámbito educativo debe ser una herramienta para comprender y abordar los problemas que la enseñanza enfrenta cada día, se deben realizar esfuerzos de integración de los saberes, en los que los sistemas aislados de conocimiento sean integrados 
de tal manera que la enseñanza sea capaz de articular el conocimiento, la contextualización y las cualidades fundamentales que el ser humano tiene, frente a la emergencia de la complejidad en las ciencias y, en particular, en la sociedad globalizada.

\section{Referencias bibliográficas}

Morin, E. (1999). Los siete saberes necesarios para la educación del futuro (Trad. Mercedes Vallejos Gómez). París, Francia: Santillana/UNESCO.

Morin, E. (2007). La cabeza bien puesta (Trad. Paula Mahler). Buenos Aires, Argentina: Ediciones Buena Visión.

Morin, E. (2002). La mente bien ordenada. (2 ${ }^{\mathrm{a}}$ ed.). Barcelona, España: Seix Barral.

Morín, E. (2004). Introducción al pensamiento complejo. México, D. F.: Editorial Gedisa. 